\title{
La « MRC une Ville » de Rouyn-Noranda : un nouveau modèle d'organisation territoriale
}

\author{
Patrice LeBlanc, Université du Québec en Abitibi-Témiscamingue \\ Denis Geoffroy, Université de Montréal
}

\section{Introduction}

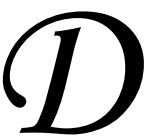
epuis quelques années, l'organisation du territoire au Québec a connu de profondes transformations. La structure municipale n'est plus ce qu'elle était il y a, par exemple, à peine cinq ans. De nombreuses fusions municipales ont eu lieu, créant plusieurs grandes villes sur le territoire du Québec, bien que quelques défusions en 2004 aient redonné leur ancien statut à certaines villes.

Un débat a également cours au Québec quant à la décentralisation des pouvoirs du gouvernement central à un niveau plus local. La création en 2002 des Conférences régionales des élus (CRÉ) en lieu et place des Conseils régionaux de développement (CRD) se voulait un premier pas vers la création d'une instance régionale à laquelle pourraient être dévolus de plus grands pouvoirs. Les maires des différentes municipalités d'un territoire - les élus - sont en effet imputables devant leur population, ce qui n'était pas le cas, selon le législateur, des représentants de la société civile qui composaient majoritairement les anciens $\mathrm{CRD}$. Si, pour certains, la décentralisation doit se faire vers une instance régionale, d'autres militent au contraire pour que ces mêmes pouvoirs soient dévolus à un niveau local, celui de la municipalité, ou à un niveau supralocal, celui de la Municipalité régionale de comté (MRC).

Dans cette double optique, celle des fusions municipales et celle du débat sur la décentralisation, le cas de la ville de Rouyn-Noranda est intéressant. En effet, ayant fusionnée en 2002 l'ensemble des municipalités du territoire de la MRC, elle est une entité locale ayant les pouvoirs d'une entité supra locale. Nous voudrions ici présenter quelques éléments d'analyse de cette nouvelle expérience. Aussi, après avoir retracé à grands traits l'histoire des fusions municipales dans la MRC de Rouyn-Noranda, nous nous attarderons à décrire l'organisation politico administrative du nouveau territoire ainsi créé. Quelques éléments de bilan termineront ce texte.

\section{L'histoire du regroupement municipal de Rouyn-Noranda}

Noranda et Rouyn reçoivent toutes deux leur lettres patentes en 1926. En 1986, après 60 ans de coexistence, elles décident de fusionner, ce qui se traduit par la naissance de Rouyn-Noranda. Par la suite, d'autres municipalités et territoires environnants viendront se joindre à ce premier noyau : Granada en 1995, Lac Dufaut en 1997, Beaudry et les territoires non organisés en 2000. C'est cependant dans la foulée du Livre blanc sur la réorganisation municipale déposé en 2000 que les choses vont changer assez radicalement sur le territoire de la MRC Rouyn-Noranda.

Dans ce Livre blanc, la ministre des Affaires municipales et de la Métropole de l'époque propose de modifier les façons de faire dans le monde municipal pour mieux soutenir les citoyens. Elle estime important d'abord d'unifier certains services municipaux nécessitant une organisation supra municipale, puis de répartir de façon équitable le fardeau fiscal et les coûts des services des municipalités et, finalement, de favoriser une conception commune et entière de l'avenir des communautés. Des regroupements municipaux sont ainsi proposés qui visent pour l'essentiel trois grands objectifs : 1) permettre aux municipalités re- 
groupées d'avoir un pôle urbain puissant et d'être économiquement concurrentielles dans un environnement de mondialisation des marchés; 2) s’assurer que l'application des priorités du gouvernement en matière d'aménagement et de développement du territoire soit respectée; 3) mettre en place une répartition plus équitable du fardeau fiscal entre les municipalités.

\section{La nouvelle ville de Rouyn-Noranda est composée de 21 quartiers, 10 en milieu urbain et 11 en milieu rural. Au niveau politique, la nouvelle ville a été divisée en 14 districts électoraux, 10 urbains (ceux de l'ancienne ville de Rouyn-Noranda) et 4 ruraux.}

En novembre 2000, la MRC de Rouyn-Noranda organise un colloque sur l'avenir des municipalités qui la composent. Plus d'une centaine de personnes d'horizons divers y participent. Les objectifs du colloque sont d'analyser les découpages territoriaux et de redéfinir le territoire des municipalités par rapport à leur viabilité. L’idée de regrouper l'ensemble des municipalités du territoire de la MRC est proposée puis acceptée par une majorité de personnes présentes, et il est demandé au gouvernement du Québec d'analyser cette proposition d' « une MRC une Ville ».

À la suite de ce colloque, au début de l'année 2001, le gouvernement du Québec nomme un mandataire pour accompagner les municipalités dans la préparation d'un rapport sur le processus de regroupement municipal de la MRC Rouyn-Noranda et, plus globalement, sur le processus lui-même de regroupement municipal. Son rapport, déposé le $1^{\mathrm{er}}$ avril 2001, contient plusieurs recommandations quant aux moyens à mettre en place sur le territoire de la MRC RouynNoranda afin de regrouper toutes ses municipalités en une seule. Cependant, les municipalités de McWatters, Évain, Mont-Brun et Destor n'appuient pas ce projet de regroupement municipal. C'est alors qu'un conciliateur du ministère des Affaires municipales et de la Métropole est nommé pour rédiger le Projet de regroupement des treize municipalités de la MRC Rouyn-Noranda, document qui fut déposé le 18 octobre 2001.

Le gouvernement du Québec rédige ensuite son propre texte et adopte, le 12 décembre 2001, un décret imposant à toutes les municipalités présentes sur le territoire de la MRC Rouyn-Noranda de se regrouper en une seule grande ville. Le premier janvier 2002, les treize municipalités de la MRC Rouyn-Noranda sont fusionnées en une seule entité administrative et un conseil municipal provisoire, composé des maires et mairesses des ancienne municipalités de la MRC Rouyn-Noranda et des conseillers de l'ancienne Ville de Rouyn-Noranda, est mis en place afin d'assurer le transfert de compétence vers la nouvelle Ville de Rouyn-Noranda ${ }^{2}$.

La population de la nouvelle ville de Rouyn-Noranda passe donc de près de 30000 citoyens à un peu plus de 40000 et occupe un territoire ${ }^{3}$ de 6435 kilomètres carrés.

\section{L'organisation politico administrative du territoire}

Juridiquement, la MRC Rouyn-Noranda n'existe plus depuis l'entrée en vigueur du décret de 2001. Selon le chapitre I article 1 de celui-ci, le nom de la nouvelle municipalité est "ville de Rouyn-Noranda ». Cependant, le chapitre II section 1 article 11 indique que « la ville est assimilée à une municipalité régionale de comté aux fins de l'application des lois suivantes : Loi sur la sécurité incendie, Loi sur les forêts, Loi sur la protection du territoire et des activités agricoles, Loi sur la qualité de l'environnement, Loi sur les terres du domaine de l'État et les articles 688 à 688.3 du Code municipal du Québec ». Avant le regroupement, les décisions concernant la gestion du territoire de la MRC Rouyn-Noranda étaient prises par le conseil des maires. Depuis l'entré en vigueur du décret, le conseil des maires de la MRC a été dissous et les pouvoirs décisionnels ont tous été transférés au conseil municipal de la nouvelle Ville de Rouyn-Noranda.

La nouvelle ville de Rouyn-Noranda est composée de 21 quartiers, 10 en milieu urbain et 11 en milieu rural. Au niveau politique, la nouvelle ville a été divisée en 14 districts électoraux, 10 urbains (ceux de l'ancienne ville de Rouyn-Noranda) et 4 ruraux. Les anciennes municipalités de D’Alembert, Cléricy, Destor et Mont-Brun forment le district nord, celles de Montbeillard, Arnthfield et Rollet sont regroupées dans le district ouest, tandis que Bellecombe, Beaudry et Cloutier composent le district sud. Les anciennes municipalités de Cadillac, Évain et McWatters ont cha- 
cun un district. Chaque district est représenté par un conseiller municipal.

Le regroupement vise à favoriser un renforcement du leadership en créant une entité plus forte. La nouvelle Ville a ainsi identifié certains principes afin de guider l'administration municipale dans son travail. Celle-ci a comme tâche de favoriser une accessibilité et une qualité de services équivalentes à tous les citoyens et citoyennes de son territoire. La Ville s'engage à assurer le soutien financier aux comités et organismes déjà soutenus par les ex-municipalités ainsi que de soutenir l'entrepreneuriat local en ayant recours aux contractants assurant déjà la prestation d'un service dans le respect des prescriptions légales. L’administration municipale doit également veiller à encourager l'implication des citoyens et des citoyennes des divers quartiers en ce qui concerne leur milieu de vie en favorisant notamment la mise en place de conseils de quartiers et en rendant disponibles les ressources nécessaires au maintien de ceux-ci.

\section{Les conseils de quartier ${ }^{4}$}

Avec la nouvelle ville de Rouyn-Noranda sont apparus sept conseils de quartier : trois en milieu urbain (Évain, McWaters et Centre-ville) et quatre en milieu rural (Cadillac, Beaudry-Bellecombe-Cloutier, Arntfield-Monbeillard-Rollet, D’Alembert-Destor-Cléricy-Mont-Brun). Ceux-ci sont des comités consultatifs créés par le conseil municipal et dont les rôles et mandats sont déterminés par celui-ci. Le conseil de quartier doit être représentatif de son milieu en y accueillant dans la mesure du possible des représentants des divers groupes actifs sur son territoire. Il doit aussi veiller au maintien de la qualité et de l'accessibilité des services dispensés à sa population et animer son milieu en jouant un rôle de forum d'échange et de lieu d'expression des besoins de sa collectivité pour alimenter le conseil municipal de la nouvelle ville. Les membres des conseils de quartiers doivent donc s'assurer de la qualité des services de proximité et de l'accessibilité des bâtiments et des équipements offerts aux citoyens du quartier. Également, ceux-ci doivent vérifier l'accessibilité des services de proximité, recommander à la Ville un soutien communautaire aux organismes du quartier et suggérer des projets afin de favoriser le développement culturel, communautaire, économique ainsi que des loisirs. Les membres recommandent également au conseil muni-

cipal les subventions que ce dernier peut accorder à des organismes du quartier.

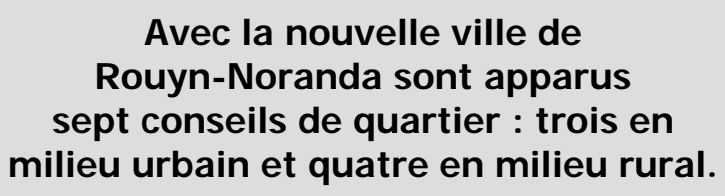

Les conseils de quartier doivent tenir un minimum de quatre rencontres annuellement et l'ordre du jour doit être publié dans un journal local en indiquant le jour, le lieu et l'heure de la rencontre. Toutes les rencontres sont publiques et tout citoyen peut intervenir dans le cadre d'une session régulière du conseil de quartier, selon les règles établies. Lors de démission, de départ ou de vacance à un siège du conseil de quartier, le conseil municipal procède à la nomination d'un nouveau membre pour combler le poste. La durée du mandat d'un membre de conseil de quartier est de quatre ans et il peut recevoir une rémunération selon la politique adopté par le conseil municipal.

Le conseil de quartier bénéficie d'un budget de fonctionnement (les salaires des coordonnateurs et les frais d'entretiens des locaux des conseils de quartier qui sont pris à même le budget de fonctionnement de la Ville) qui est accordé par le conseil municipal. En février 2004, le conseil municipal a déterminé que les revenus de location ou de prêt des salles communautaires d'un quartier seront dévolus au conseil de quartier pour répartition pour la vie de quartier. Cette mesure vise l'amélioration des services offerts aux citoyens. Finalement, les activités suivantes : dons et subventions, activités récréatives, parcs, patinoires, journaux de quartier ont été décentralisées aux quartiers et les bibliothèques et immeubles communautaires ont été déconcentrées.

\section{Les services de proximité}

En février 2002, le conseil municipal provisoire met en place un comité composé de cinq élus et de quatre administrateurs pour soumettre une proposition et des modalités de mise en place des services de proximité ${ }^{5}$ sur le territoire de la nouvelle ville de RouynNoranda. Trois modes de desserte sont alors proposés : 1) certains services devraient être centralisés au bureau du responsable du service qui sera donc appelé périodiquement à aller intervenir sur le territoire; 2) 
d'autres services devraient être déconcentrés près du lieu de résidence des citoyens, obligeant ainsi les fonctionnaires attitrés à ce service à établir un horaire de présence dans les divers quartiers de la ville; 3) enfin, d'autres services devraient être décentralisés dans les quartiers ou districts et dispensés par des employés permanents relevant d'un responsable à la Ville de Rouyn-Noranda. Le comité propose également la création de douze postes d'agents d'animation et de développement du milieu dans les quartiers ruraux de la ville. Leurs principales fonctions auraient été de soutenir les travaux des conseils de quartier, de faire office de vigie locale et d'animer et de développer le milieu.

En mai 2003, trois nouveaux types de poste à la Ville de Rouyn-Noranda ont été créés afin d'assurer les services de proximité dans ses différents quartiers. Il s'agit des fonctions de coordonnateur de service de proximité et développement rural (décentralisé), de préposé aux permis et vigie de quartier (décentralisé) et de contremaître de secteur (déconcentré). La création des trois fonctions a généré l'embauche de six coordonnateurs de service de proximité et développement rural, six préposés à la réglementation et à la vigie locale répartis dans les districts nord, sud, est, ouest ainsi que dans les quartiers McWaters et Évain. Également, deux contremaîtres de secteur pour la voirie et les travaux publics et un contremaître aux immeubles, dédié à $50 \%$ au territoire rural de la ville, ont été engagés.

\section{Depuis la mise en application du projet de loi 34, le CLD relève plus directement de la Ville de Rouyn-Noranda.}

\section{Les comités}

Cinq comités existaient au sein de la MRC RouynNoranda avant le regroupement. Le comité consultatif agricole (CCAG), entité obligatoire selon la loi, a été maintenu et son fonctionnement est demeuré identique. Le comité consultatif en aménagement s'est mué en une commission d'aménagement au sein de la nouvelle ville et son fonctionnement est resté semblable. Le comité consultatif de sécurité publique a quant à lui été transformé en une commission de la sécurité publique au sein de la nouvelle ville et rien n'a changé du point de vue de son travail. Le comité multi ressource a gardé les mêmes fonctions avec le regroupement. Finalement, l'instance décisionnelle de la MRC Rouyn-Noranda, soit les 13 maires de ses municipalités rurales et celui de Rouyn-Noranda, a laissé sa place au conseil municipal de la nouvelle ville composé de 14 conseillers et du maire.

\section{Le développement local}

La Ville de Rouyn-Noranda est soucieuse du développement économique et social de son territoire. Elle a à sa disposition deux leviers qu'il vaut la peine ici d'examiner.

\section{Le Centre local de développement}

En 1974, la Commission industrielle de RouynNoranda voit le jour et est gérée par la Ville de Rouyn-Noranda. Quatre ans plus tard, en 1978, à la suite de restructurations, la Commission industrielle devient la Corporation de développement industriel et commercial du Rouyn-Noranda régional (CDICRN), tandis qu'en 1987, la CDICRN est transformée en Société de développement économique de RouynNoranda (SDE-RN). En 1993, le champ d'action de la SDE-RN s'élargit à l'ensemble du territoire de la MRC Rouyn-Noranda. En 1998, dans la foulé de la création des CLD par le gouvernement québécois, la SDE-RN est remplacée par le Centre local de développement de Rouyn-Noranda (CLD-RN). Le nombre d'administrateurs de la SDE-RN, qui était de 15 depuis 1993, reste identique jusqu'en 2005, année où elle se voit obligée d'en augmenter le nombre à 21 afin de se conformer aux nouvelles dispositions du projet de loi $34^{6}$.

Au temps de la SDE-RN, les protocoles d'ententes, notamment quant à son financement, étaient signés avec la MRC-RN et le gouvernement du Québec. Cela s'est poursuivi après sa transformation en CLD, tandis que, lorsque la MRC Rouyn-Noranda est devenu " une Ville une MRC » en 2002, le CLD-RN a signé sont entente avec la Ville et le gouvernement du Québec. Par contre, depuis la mise en application du projet de loi 34, le CLD relève plus directement de la Ville de Rouyn-Noranda. En effet, celle-ci signe d'abord un protocole avec le gouvernement du Québec et, par la suite, le CLD-RN signe son protocole d'entente avec elle.

En 2004, le CLD a investi près de 1160000 \$ dans des entreprises locales pour générer des investisse- 
ments totaux de 6,3 M\$. Une cinquantaine d'entrepreneurs ont réalisé leur projet d'affaires, que ce soit au niveau d'un démarrage, d'une acquisition ou d'une expansion, et ils ont créé 64,5 nouveaux emplois tandis que 112,5 autres ont été maintenus. Quoique la majorité des entreprises concernées œuvrent dans le secteur des commerces et services, les secteurs industriel et de la nouvelle technologie ont également été très actifs ${ }^{7}$.

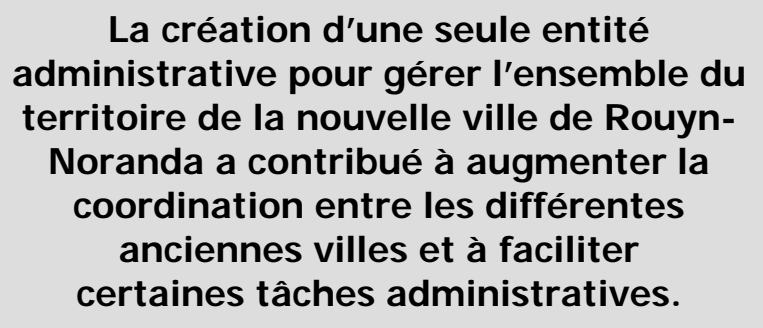

Le Pacte rural

Dans le cadre de la Politique nationale de la ruralité, la Ville de Rouyn-Noranda ${ }^{8}$ a signé, le 25 mars 2003, le Pacte rural avec le gouvernement du Québec. Ce pacte vise à promouvoir le développement du milieu rural en fonction des trois orientations de la Politique nationale de la ruralité, soit de stimuler et soutenir le développement durable et la prospérité des collectivités rurales, d'assurer la qualité de vie de ces collectivités et de renforcer leur pouvoir d'attraction, ainsi que de soutenir l'engagement des citoyens envers le développement de leur communauté. Les onze quartiers ${ }^{9}$ ruraux de la nouvelle ville de Rouyn-Noranda composent le territoire d'application du Pacte rural. Mais si c'est la ville qui signe l'entente, c'est cependant le CLD-RN qui se voit confier les responsabilités relatives au mandat prévu à la Loi sur le ministère des Régions.

Dans ce cadre, la Ville de Rouyn-Noranda reçoit du gouvernement une aide financière de 627737 \$ pour une période de cinq ans. Un addenda au Pacte rural prévoit de plus que le gouvernement versera un montant supplémentaire de 20000 \$ par année pendant les cinq années du pacte pour l'engagement d'un agent de développement rural, dont une partie ou la totalité des tâches sera d'appuyer le mise en œuvre du Pacte rural. La Ville s'engage quant à elle à une contribution au moins équivalente. Si, ailleurs au Québec, c'est l'agent rural du CLD qui doit appuyer la mise en œuvre du Pacte, ici l'agent peut travailler, au choix de la Ville, au sein du CLD ou de la Ville. Pour l'instant, une coordonnatrice du développement rural ainsi que quatre agents ruraux travaillant dans les quartiers ruraux de la ville et payés à l'extérieur de l'enveloppe budgétaire original du Pacte sont à l'emploi du CLD.

En mai et juin 2004, une Corvée rurale s’est déroulée dans les onze quartiers ruraux de la ville de RouynNoranda afin de connaître leurs besoins et leurs enjeux actuels. Cette vaste consultation publique, qui avait été précédée de portraits socio-économiques et de portraits portant sur la dynamique communautaire des différents quartiers, a permis d'identifier des orientations prioritaires de développement. Par la suite, deux appels d'offres à projets ont été lancés, donnant lieu au financement de six projets en 2004 et de onze en 2005.

\section{Bilan préliminaire}

Après quatre ans de fonctionnement, quel bilan peuton faire de cette nouvelle structure municipale ? Nous examinerons cette question sous trois angles : celui de la gestion et de la coordination des différentes activités de la ville, celui des conseils de quartier et, finalement, celui du développement économique.

\section{Gestion et coordination quotidiennes des activités de la Ville}

La création d'une seule entité administrative pour gérer l'ensemble du territoire de la nouvelle ville de Rouyn-Noranda a contribué à augmenter la coordination entre les différentes anciennes villes et à faciliter certaines tâches administratives. Le cas du service des forêts est assez probant à cet égard. Auparavant, à la MRC Rouyn-Noranda, le service devait obtenir l'approbation de quatorze signataires pour la convention d'aménagement et autres ententes. Dans les faits, cela voulait dire que les quatorze maires devaient s'entendre avec leur conseil municipal respectif avant de signer une entente et cela causait, à l'occasion, beaucoup de retard dans l'échéancier des services offert aux citoyens. Aujourd'hui, nous retrouvons une seule entité signataire, le conseil municipal de la Ville de Rouyn-Noranda, ce qui, dans les faits, facilite la prise de décisions et rend plus opérationnel le service aux citoyens. Le regroupement municipal a également amené une plus grande spécialisation des ressources 
humaines dans chacun des secteurs d'activités de la nouvelle ville, ce qui, pour plusieurs intervenants, se traduit par une meilleure gestion administrative pour les citoyens.

Cependant, le regroupement municipal a entraîné avec lui une plus grande complexité administrative. En effet, la fusion des différentes municipalités était aussi dans les faits la rencontre de l'urbain et du rural, la rencontre donc de deux cultures administratives ainsi que de deux cultures territoriales. La MRC Rouyn-Noranda et les municipalités rurales étaient régies par le Code municipal, tandis que la ville de Rouyn-Noranda est régie par la Loi sur les cités et villes. L'harmonisation des différents règlements, devant aussi bien s'appliquer en milieu urbain que rural, n’est pas toujours aisée.

Certains aspects reliés au financement de la nouvelle ville de Rouyn-Noranda posent également quelques problèmes. Avant les fusions municipales, les petites municipalités environnantes recevaient, de par leur petite taille, des subventions auxquelles la grande ville de Rouyn-Noranda n’a plus accès. Le partage de la taxe sur l'essence illustre bien cette situation. La nouvelle ville de Rouyn-Noranda ne pourra pas bénéficier du partage plus élevé par habitant qu'auraient reçu les petites villes de moins de 6500 habitants. S'il n'y avait pas eu de regroupement municipal l'ensemble des municipalités de la MRC Rouyn-Noranda aurait en fait reçu plus d'argent que la ville fusionnée en recevra. Il semble également que certaines subventions, notamment pour des travaux de la voirie et des infrastructures, autrefois dédiées et reliées à la MRC, seraient en régression.

\section{Conseils de quartier : une expérience mitigée}

La mise sur pied de conseils de quartier est sans doute une des transformations les plus importantes de l'organisation politico administrative du territoire de Rouyn-Noranda. Dans une certaine mesure, ces conseils de quartier devaient, en milieu rural, remplacer les anciennes mairies. Malgré quelques succès, les résultats ne sont cependant pas toujours à la hauteur des attentes des citoyens et des intervenants municipaux.

Parmi les dix quartiers dit urbains, seulement deux conseils de quartiers ont été créés, soit ceux d’Évain et de McWatters. Il y a bien aussi un conseil de quartier dans le district du centre-ville, mais celui-ci n’a tenu que deux réunions depuis sa création. Dès la deuxième rencontre, il n'y avait pas quorum et, par la suite, toutes les tentatives du conseiller du centre-ville d'effectuer une rencontre ont été vaines. Si, dans les faits, le conseil de quartier existe toujours, il est incapable de tenir une réunion par manque de membres.

\section{Malgré quelques succès, les résultats ne sont cependant pas toujours à la hauteur des attentes des citoyens et des intervenants municipaux.}

Pour certaines personnes, autant le conseil municipal de la Ville de Rouyn-Noranda que les anciens conseils municipaux des petites municipalités étaient plus conviviaux avant le regroupement. Depuis le regroupement et la mise en place des conseils de quartier, les citoyens des anciennes municipalités ont perdu une place importante d'échange et de discussion pouvant mener à une décision locale. Pour ceux-ci, les conseils de quartier sont purement consultatifs, faisant par le fait même disparaître un pouvoir décisionnel local. Cela n'indique rien de bon pour l'avenir des anciennes communautés ${ }^{10}$.

En 2004, une étude ${ }^{11}$ a d'ailleurs démontré que le pouvoir des conseils de quartier est à la baisse. L'effet de la fusion sur les anciens conseils municipaux et sur les citoyens est la centralisation du pouvoir au sein d'un seul conseil municipal regroupant treize anciennes municipalités rurales. Les citoyens trouvent « difficile l'exercice quotidien de cette nouvelle configuration politique [...] [et] pensent que ce n'est pas d'en haut que les choses peuvent se faire et se désolent que ce soit des urbains qui gèrent du rural ». Également, à la suite du regroupement, certaines anciennes municipalités se retrouvent à plus de 50 kilomètres du lieu décisionnel. Les citoyens trouvent difficile «d'accepter que des décisions qui les concernent soient prises par des gens qui résident aussi loin d'eux ». Ainsi, certains citoyens ont « le sentiment que la responsabilité réelle du conseil de quartier échappe parfois au contrôle local ».

La Ville de Rouyn-Noranda a tenue le 9 avril 2005 une journée de rassemblement des conseils de quartier sous le thème "Est-ce que les conseils de quartier permettent l'engagement citoyen, et de relever le défi du développement » à laquelle 58 personnes ont participé. La question des communications a été l'un des 
aspects les plus discutés lors de cette journée. Les participants ont souligné dans une large proportion l'importance de faire connaître davantage auprès de la population et des conseils de quartier les outils en place, les rôles de chacun, les sources d'aide disponibles sur le territoire. Il a également été mentionné qu'il y a un manque de clarté dans le mandat, la mission et les cadres de fonctionnement des conseils de quartier. Plusieurs ont souhaité l'amélioration des réseaux de communication entre les différents intervenants sur le terrain tels que les conseils de quartier, le conseil municipal, les agents de développement et les employés des divers services municipaux. Selon plusieurs participants, les conseils de quartier devraient cibler et rencontrer la population locale pour lancer des idées, des rêves afin d'établir une communication entre leurs membres et la population. Cela pourrait avoir un effet de synergie et susciter l'intérêt à participer aux rencontres du conseil de quartier, créant ou recréant une dynamique communautaire sur le territoire.

Les participants ont également indiqué que le conseil de quartier doit être mobilisateur et créer un rapprochement entre les différents organismes de leur territoire. Certains ont mentionné que la tenue de réunions mensuelles permettrait d'augmenter la motivation et la communication au sein de la population. Également, afin de favoriser l'implication des membres d'un conseil de quartier, ceux-ci devraient avoir un dossier particulier et des responsabilités dans un domaine précis. Dans une large proportion, les participants souhaiteraient même se voir confier des responsabilités accrues de la part du conseil municipal. Certains participants ont indiqué que les conseils de quartier pourraient jouer un rôle de comité de développement et alors élaborer un plan de développement par quartier. Plusieurs souhaitent aussi que tous les quartiers se dotent d'un agent de développement afin de faire progresser les projets et de maintenir le rôle d'animation du milieu qui est essentiel.

\section{Le développement économique}

$\mathrm{Si}$, auparavant, il pouvait parfois exister une certaine compétition entre les petites municipalités concernant leur développement économique et celui de la MRC, cela est un peu moins vrai maintenant. En effet, le conseil municipal de la nouvelle ville peut plus facilement effectuer une planification économique globale et développer un point de vue commun sur la fis- calité de la nouvelle ville. De plus, elle peut plus aisément que les anciennes municipalités avoir accès à des sources de financement plus importantes pour effectuer certains travaux.

Au niveau du CLD, la nouvelle organisation municipale fait en sorte qu'il relève de la Ville et qu'il ne peut donc plus négocier directement avec le gouvernement du Québec pour son financement. Actuellement, ce sont les élus qui font cette négociation budgétaire, élus que certains estiment moins proches du développement économique que les administrateurs du CLD. Cette situation pourrait donc nuire à son financement. Par ailleurs, avant 2002, le CLD-RN avait une réunion par année avec le conseil municipal de Rouyn-Noranda pour exposer ses travaux. Paradoxalement, actuellement, il n'a plus de temps de présentation auprès du conseil municipal pour expliquer et faire valoir ses interventions aux membres du conseil municipal.

Enfin, toujours au niveau du développement économique, certains citoyens ont indiqué, lors de la journée de rassemblement d'avril 2005, que les demandes provenant du Pacte rural devraient être traitées avec autant d'importance que les demandes provenant de l'urbain, car pour certains, les délais pour recevoir les réponses sont trop longs.

\section{Le conseil municipal de la nouvelle ville peut plus facilement effectuer une planification économique globale et développer un point de vue commun sur la fiscalité de la nouvelle ville.}

\section{Conclusion}

La forme municipale « une MRC une Ville » peut-elle être un modèle d'organisation politico administrative et un lieu de décentralisation des pouvoirs de l'État ? Bien qu'elle soit encore quelque chose de relativement récent dans le paysage québécois, deux éléments retiennent tout de même plus particulièrement l'attention. L'organisation politique de ce territoire fait en sorte que nous sommes en présence d'une MRC (son équivalent en fait) avec un " préfet » élu au suffrage universel (plus précisément le maire) et un pouvoir direct de taxation. Ce modèle nous semble être porteur, comme nous l'avons vu, d'une coordination plus forte des différents acteurs du milieu et d'une capacité 
plus grande de penser d'une façon plus globale le développement du territoire. De plus, le niveau d'imputabilité du maire-préfet est plus élevé dans la nouvelle ville de Rouyn-Noranda qu'il ne l'était auparavant. Dans une perspective de décentralisation de pouvoirs de l'État central, cette configuration locale est intéressante.

La mise sur pied des conseils de quartier est l'autre élément intéressant de cette réorganisation territoriale. Le bilan est cependant ici un peu moins reluisant. Les conseils de quartier sont encore dans la phase d'implantation, et le processus de regroupement municipal que les citoyens de Rouyn-Noranda ont connu au cours des quatre dernières années a été très difficile pour les anciens résidents des municipalités regroupées. Le regroupement a encore des effets de démotivation sur les conseils de quartier et sur la population en général. Le modèle semble aussi avoir de la difficulté à s’implanter en milieu urbain. Le pouvoir qui était réparti auparavant à travers treize conseils municipaux est dorénavant centralisé au seul conseil municipal de la nouvelle ville de Rouyn-Noranda. Une grande majorité de citoyens ruraux ont le sentiment d'avoir perdu du pouvoir au profit d'une entité qui les représente peu. Nous pouvons observer un certain désengagement de la part des citoyens à la vie sociocommunautaire et, surtout, pour un nombre relativement important de citoyens, une perte du sentiment d'appartenance à leur municipalité depuis le regroupement.

L'analyse qui précède nous semble déboucher sur deux défis importants qu'aura à relever la forme « une MRC une Ville»: celui de la conciliation rural/urbain et celui de la démocratie locale et de la participation des citoyens. On l'a bien vu, le regroupement municipal qui a donné naissance à la nouvelle ville de Rouyn-Noranda en 2002 a obligé à la cohabitation deux modes de vie forts différents, le rural et l'urbain, peu habitués de gouverner ensemble au sein d'une même entité administrative. Des apprentissages ont sans doute été faits quant à cette nécessaire cogestion des territoires, mais du chemin reste encore à parcourir. De plus, si, pour l'instant, un certain équilibre existe au sein du conseil municipal entre les représentants élus des territoires ruraux et urbains de la ville, une reconfiguration des districts électoraux sur la base de la population pourrait venir le briser et isoler les parties rurales de la ville.
La création d' " une MRC une Ville » fait également en sorte d'engendrer une très grande ville d'un strict point de vue géographique. Plusieurs citoyens des quartiers ruraux se sentent maintenant bien loin des lieux de décision. La participation aux réunions du conseil municipal devient difficile, les déplacement pour y assister étant souvent trop longs, voire dangereux, notamment en hiver. Les conseils de quartier devaient pallier ce problème. À l'expérience, ils n'ont pas encore totalement donné les bonnes réponses. La mise en place des conseils de quartiers a en effet donné lieu à plusieurs mécontentements au sein de la population rurale, notamment parce que, pour certains citoyens, les conseils de quartier n'ont aucun pouvoir politique et donc aucun moyen d'intervenir ou d'agir sur leur propre territoire. Rappelons à cet effet que les membres des conseils de quartier ne sont pas élus démocratiquement mais bien nommés par le conseil de Ville. Il reste donc encore des ajustements à apporter à cette structure pour qu'elle devienne réellement un lieu de participation démocratique des citoyens à leur cité.

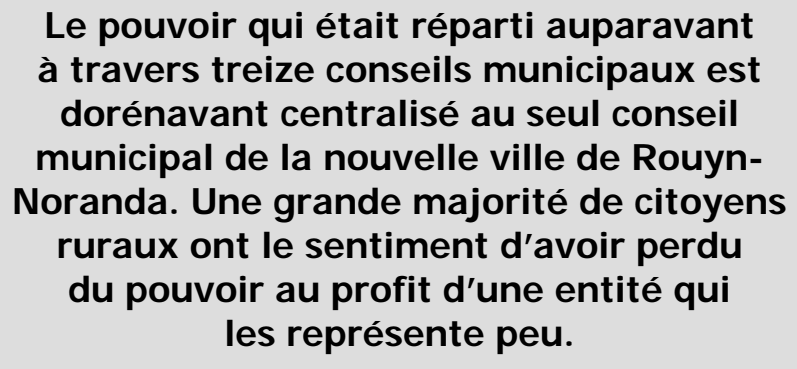

En définitive, le regroupement municipal de la ville de Rouyn-Noranda est relativement jeune. L'exemple " une MRC une Ville » de Rouyn-Noranda est certes intéressant et pourrait servir de modèle à d'autres réorganisations municipales au Québec. Néanmoins, il reste encore du travail à effectuer afin d'en arriver à une nouvelle forme d'organisation municipale adéquate et adaptée aux citoyens, plus particulièrement ceux vivant en milieu rural.

\section{Notes et références}

1 Gouvernement du Québec, Ministère des Affaires municipales et de la Métropole (2000). La réorganisation municipale : changer les façons de faire pour mieux servir les citoyens, Livre blanc sur la réforme municipale, Québec, MAMM, 35 p. 
2 Sur l'histoire récente des fusions municipales à RouynNoranda on lira : Ayotte, M. (2003), «L'influence de la perte des gouvernements locaux, suite aux fusions municipales, sur la vitalité culturelle et communautaire des petites collectivités rurales », mémoire de maîtrise, Université du Québec en Abitibi-Témiscamingue.

3 Rouyn-Noranda devient ainsi la $20^{\mathrm{e}}$ ville en importance au Québec. Elle a une population de taille semblable à celle de Rimouski, mais avec un territoire 25 fois plus grand.

4 Ville de Rouyn-Noranda. (2002). La mise en place des conseils de quartier : document d'information générale, Rouyn-Noranda, 19 p.

5 Sont considérés comme des services de proximité les services municipaux suivants: Les services rendus directement aux citoyens : l'émission des permis, le paiement des taxes et des amendes, l'échange d'information et le traitement des plaintes. Les services concernant les immeubles, tels l'entretien des rues, la desserte en aqueduc et égout, la gestion des matières résiduelles, la protection incendie, la sécurité publique, l'éclairage de rue, l'évaluation foncière, l'urbanisme et le zonage. Les services reliés à la collectivité, tels l'organisation d'activités de loisir et de culture, la bibliothèque municipale locale, l'entretien du cimetière, les parcs et terrains de jeux, le soutien aux organismes communautaires locaux ainsi qu'au développement communautaire local.
6 Loi sur le ministère du Développement économique et régional et de la Recherche.

7 CLD-RN. (2005), « 2004, une année fertile pour le CLD de Rouyn-Noranda », communiqué de presse diffusé le 31 janvier 2005.

8 Ailleurs au Québec, cette entente est plutôt signée entre les MRC et le gouvernement du Québec.

9 Ce sont en fait les territoires des anciennes municipalités et territoires non organisés de Arntfield, Beaudry, Bellecombe, Cadillac, Cléricy, Cloutier, D’Alembert, Destor, Lac-Dufault, Lac-Montanier, Lac-Surimau, McWatters, Montbeillard, Mont-Brun, Rapide-des-Cèdres et Rollet (Ville de Rouyn-Noranda, 2003).

10 Cette analyse doit cependant être mise en perspective avec le fait qu'avant que le regroupement ait lieu, il y avait déjà un certain désintéressement des citoyens des petites municipalités face à leur conseil municipal et que plusieurs petites municipalités avaient de la difficulté à recruter des conseillers municipaux.

11 Agence de développement de réseaux locaux de services de santé et de services sociaux de l'Abitibi-Témiscamingue, (ADRSSSSAT). (2004). La dynamique communautaire dans les quartiers ruraux de la ville de Rouyn-Noranda, $78 \mathrm{p}$. 


\section{Publicité}

\section{Revue OT}

Site Web 